\title{
CMOS Integrated Chemical Microsensors And Their Circuitry
}

Key words

CMOS sensors, Microsensors, Chemical sensors, Sensorinterface circuitry, Chemical microsensors
Henry Baltes

Oliver Brand

Christoph Hagleitner

Physical Electronics Laboratory

\section{INTRODUCTION}

Chemical sensors and electronic noses are used nowadays in a variety of industrial and environmental applications, such as online process monitoring in the food-industry and personal safety by threshold limit value (TLV) monitoring. Commercially available desktop-size electronic noses (e.g., MOSES II [1]) are based on arrays of chemical sensors utilizing different chemically sensitive films for selectivity enhancement. Sensing structures include, e.g., quartz crystal microbalances, surface acoustic wave devices, calorimetric sensors, and metal-oxide sensors.

In recent years, industrial CMOS and BiCMOS processes in combination with silicon micromachining have been used for the microfabrication of a variety of sensors, such as infrared radiation detectors, flow sensors, and pressure sensors $[2,3,4,5]$. The sensors benefit from the reliability of well established IC processes and the cointegration of signal conditioning circuitry.

In this paper, we review CMOS chemical microsystems developed at the Physical Electronics Laboratory of ETH Zurich for detection of VOCs in air. Prototypes based on three different sensing principles are presented: (i) capacitive, (ii) gravimetric or resonant, and (iii) calorimetric chemical microsensors. The emphasis of the paper is on the signal conditioning circuitry cointegrated with the different sensing structures. The combination of arrays of these sensors with different chemically sensitive polymer films leads to the development of hand-held application specific electronic micronoses [6].

\section{MICROSENSOR FABRICATION}

The chemical microsensors presented in this paper are fabricated using an industrial $0.8 \mu \mathrm{m}$ CMOS process from Austria Mikro Systeme International (Unterpremstätten, Austria) in combination with post-CMOS micromachining and polymer deposition. After completion of the CMOS process, silicon membranes and suspended $n$-well structures are released by anisotropic etching from the back of the wafer using a potassium hydroxide $(\mathrm{KOH})$ solution in combination with an electrochemical etch-stop technique. The etching stops at the pn-junction between the CMOS nwell and the p-type substrate. The special preparation sequence for the electrochemical etching of CMOS wafers is described in [7] and is available as a foundry service from Austria Mikro Systeme (AMS).

The electrochemical etch-stop technique provides silicon-based microstructures that benefit from the excellent mechanical properties of silicon. Moreover, active devices can be designed within the n-well structures. By combining the described bulk-micromachining process with additional reactive-ion-etching (RIE), not only membrane structures, but also bridges and cantilever beams can be fabricated.

CMOS Integrated Chemical Microsensors And Their Circuitry.

By Henry Baltes, Oliver Brand, Christoph Hagleitner (Physical

Electronics Laborator). E-mail : baltes@iqe.phys.ethz.ch

\section{CMOS Chemical Microsensors}

After completion of the post-CMOS micromachining steps, polymer films acting as chemically sensitive layers are deposited onto the sensing structures by spray-coating through a shadow mask. Judicial choice of the chemically sensitive polymer layers provides a trade-off between reversibility of the polymer-analyte interaction and sensitivity towards certain analytes.

The microsensor dice are mounted in standard 28-pin ceramic dual-in-line packages for testing. A flip-chip packaging technique for the chemical microsystems has also been demonstrated [8]. For characterization, the dual-in-line packages containing the CMOS gas sensors are mounted onto the measurement chamber of a computer-controlled flow setup [9].

\subsection{Capacitive Microsensors}

In the case of capacitive chemical microsensors, the change of the dielectric constant of the polymer upon absorption of analyte is sensed. To this end, interdigitated capacitor structures are formed by the two metal layers of the CMOS process [8]. In order to enable measurements at different temperatures, the sensor and reference capacitors are placed on thermally insulated island struc-

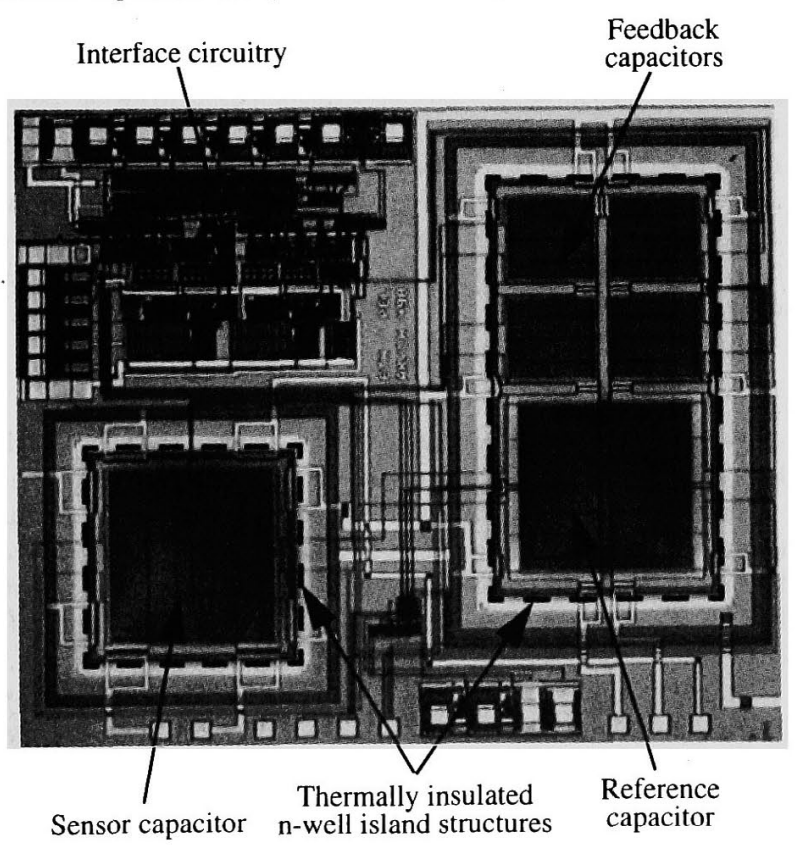

Figure 1. Photograph of the capacitive chemical microsystem. The interdigitated capacitors are located on thermally insulated $n$ well islands to allow measurements at different temperatures [10]. 
tures (micro hotplates) released using anisotropic etching with electrochemical etch-stop [10]. On-chip heating resistors consisting of the gate polysilicon layer of the CMOS process are located on the island structures. The temperature is measured by pn-diodes integrated in every n-well island. A photograph of the complete capacitive chemical microsystem chip is shown in Fig. 1.

On-chip readout circuitry converts the difference between the polymer coated sensing and a reference capacitance into a digital output signal. This is done by incorporating both capacitors in the first stage of a fully differential second order switched capacitor $\Sigma \Delta$-modulator [11]. The converter provides a pulse density modulated output that can be decimated using a frequency counter.

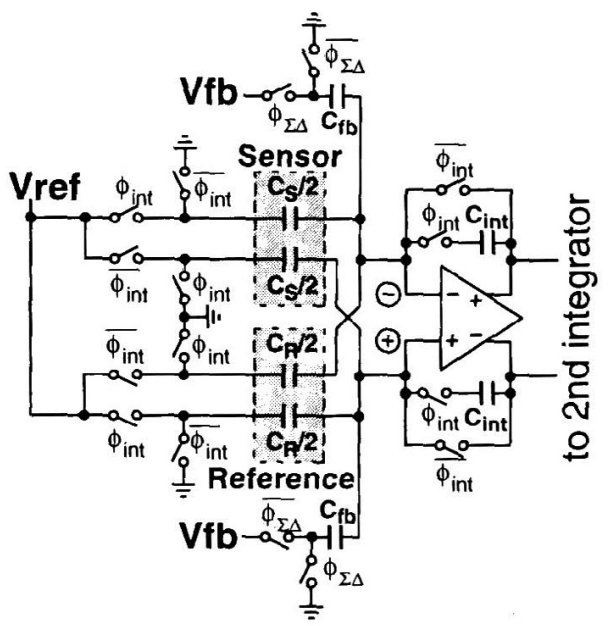

Figure 2. First stage of second order switched capacitor $\Sigma \Delta$ modulator. Sensor and reference capacitors are split in two parts to improve the charge transfer efficiency [10].

Fig. 2 shows the first stage of the $\Sigma \Delta$-modulator. With $V_{\text {ref }}=V_{\mathrm{fb}}$ [11] and given the small capacitance changes due to analyte absorption, the converter uses only a fraction of its dynamic range. By operating the first stage of the $\Sigma \Delta$-modulator at a higher clock frequency compared to the rest of the converter, the signal can be amplified. With every clock cycle $\Phi_{\text {int }}$, the charge $V_{\text {ref }}{ }^{*}\left(C_{S}-C_{R}\right)$ is accumulated on the integrating capacitor $\mathrm{C}_{\text {int }}$. If the conversion takes place at a $2^{n}$ times lower clock frequency $\Phi_{\Sigma \Delta}$, the output frequency is given by [10]

$$
f_{\text {out }}=\frac{f_{S}}{2}\left(1+2^{n} \frac{C_{S}-C_{R}}{C_{f b}}\right)
$$

with $C_{S}, C_{R}$, and $C_{f b}$ denoting the sensor, reference, and feedback capacitance and $\mathrm{f}_{\mathrm{s}}$ the sampling frequency.

In order to minimize the sensitivity of the output signal towards temperature variations and aging, all feedback capacitors required in the circuit are realized as interdigitated capacitors and are located on a thermally insulated $n$-well island together with the reference capacitor.

After dicing, the sensing capacitors were spray-coated with a $10 \mu \mathrm{m}$ poly(etherurethane) (PEUT) layer while the reference and feedback capacitors were covered with a $10 \mu \mathrm{m}$ poly(dimethylsiloxane) (PDMS) layer. The limit of detection of the capacitive microsystem at $28{ }^{\circ} \mathrm{C}$ for toluene and ethanol was measured to be $8 \mathrm{ppm}$ and $5 \mathrm{ppm}$, respectively [10].

Fig. 3 shows the measured frequency shift for 4250 ppm of ethanol and $2600 \mathrm{ppm}$ of toluene at different sensor temperatures. The measured absolute frequency shift for a single analyte decreases exponentially with temperature because of the temperature depen- dence of the partition coefficient. Different exponential factors of the sensor responses for ethanol and toluene reflect the different temperature dependences of the partition coefficients. Thus, the temperature dependent output signal can distinguish between different analytes. The frequency shifts for a mixture of analytes can be calculated by adding the signals from the single analytes [10].

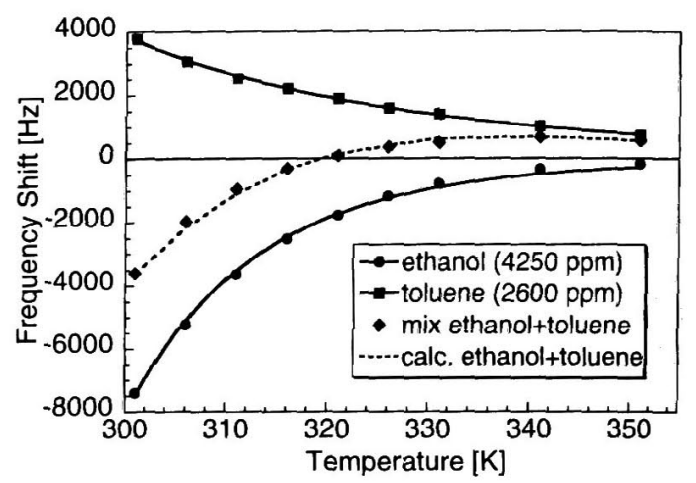

Figure 3. Measured frequency shift of the capacitive chemical microsystem as a function of the sensor temperature for $4250 \mathrm{ppm}$ ethanol, $2600 \mathrm{ppm}$ toluene, and a mixture of $4250 \mathrm{ppm}$ ethanol and 2600 ppm toluene [10].

\subsection{RESONANT MICROSENSORS}

Resonant or gravimetric chemical sensors sense the mass change of the chemically sensitive polymer layer upon absorption of analyte molecules. A CMOS resonant chemical sensor based on a $150 \mu \mathrm{m}$ long silicon cantilever beam is shown in Fig. 4 [12].

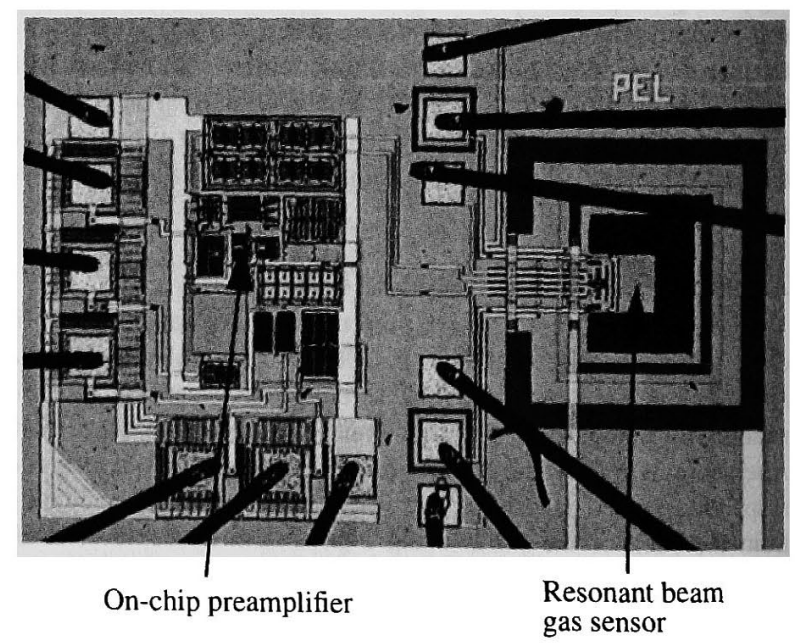

Figure 4. Micrograph of a 150 um long resonant beam gas sensor connected to an on-chip preamplifier [12].

The beam consists of the silicon n-well covered by the dielectric layers of the CMOS process. The cantilever structures are released by anisotropic etching with an electrochemical etch-stop technique and two additional reactive-ion-etching steps. After micromachining, the cantilevers are spray-coated with the chemically sensitive polymer film. The cantilever beams feature resistors for electrothermal excitation and piezoresistive detection of transverse vibrations. The four diffused $\mathrm{p}^{+}$-piezoresistors are arranged in a Wheatstone bridge configuration. An on-chip low-noise differ- 
ential difference amplifier (DDA) as shown in Fig. 5 is used to amplify the weak signals from the Wheatstone bridge and eliminate the capacitive crosstalk observed with off-chip amplification.

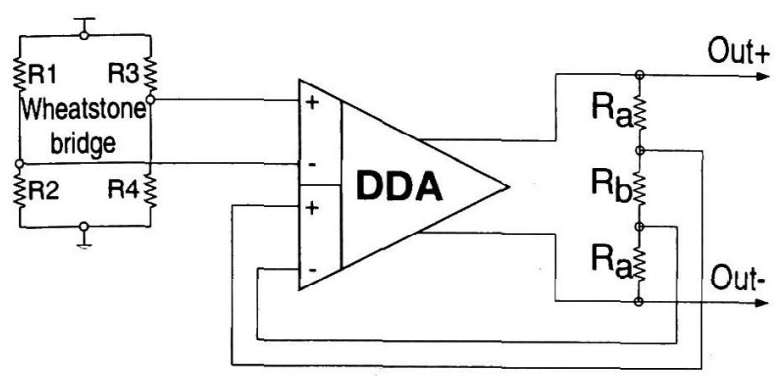

Figure 5. Block diagram of differential difference amplifier cointegrated with the gravimetric chemical microsystem.

In contrast to an operational amplifier, the DDA amplifies the difference between its differential input voltages. The gain is defined by the feedback resistors $R_{\mathrm{a}}$ and $\mathrm{R}_{\mathrm{b}}$ :

$$
A_{v}=1+\frac{R_{a}}{R_{b}}
$$

Compared to the standard instrumentation amplifier configuration with two operational amplifiers, the DDA offers a reduction in circuit complexity and an independent choice of the output common mode voltage.

The gravimetric chemical sensor is operated at its fundamental resonance frequency using an external feedback loop [12]. To this end, the output signal of the DDA is limited by a comparator and then applied to the heating resistors. The performance of the DDA and feedback system are summarized in Table 1.

\begin{tabular}{|c|c|}
\hline \multicolumn{2}{|c|}{ Differential Difference Amplifier (DDA) } \\
\hline Gain & $30 \mathrm{~dB}$ \\
\hline 3- $d B$ bandwidth $\mathrm{f}_{3 \mathrm{~dB}}$ & $500 \mathrm{kHz}$ \\
\hline Equivalent input noise & $15 \mathrm{nV} / \sqrt{\mathrm{Hz}}$ \\
\hline \multicolumn{2}{|c|}{ Feedback Loop } \\
\hline Fundamental frequency & $354 \mathrm{kHz}$ \\
\hline $\begin{array}{c}\text { Allan variance } \\
\text { (short time stability) }\end{array}$ & $0.03 \mathrm{~Hz}$ \\
\hline
\end{tabular}

Table 1. Performance of differential difference amplifier cointegrated with the resonant gas sensor and stability of the feedback loop.

The linear response of the resonant gas sensor coated with a $2.4 \mu \mathrm{m}$ poly(etherurethane) (PEUT) layer to the analyte concentration is shown in Fig. 6 for different analytes. Assuming a minimal detectable frequency shift of $1 \mathrm{~Hz}$, the limit of detection (LOD) is less than $10 \mathrm{ppm}$ for toluene and $70 \mathrm{ppm}$ for ethanol [12].

\subsection{Calorimetric Microsensors}

The calorimetric chemical sensor measures the enthalpy change upon absorption and desorption of analyte molecules into the chemically sensitive polymer. The CMOS calorimetric gas sensor shown in Fig. 7 consists of a $595 \mu \mathrm{m}$ by $2162 \mu \mathrm{m}$ suspended n-

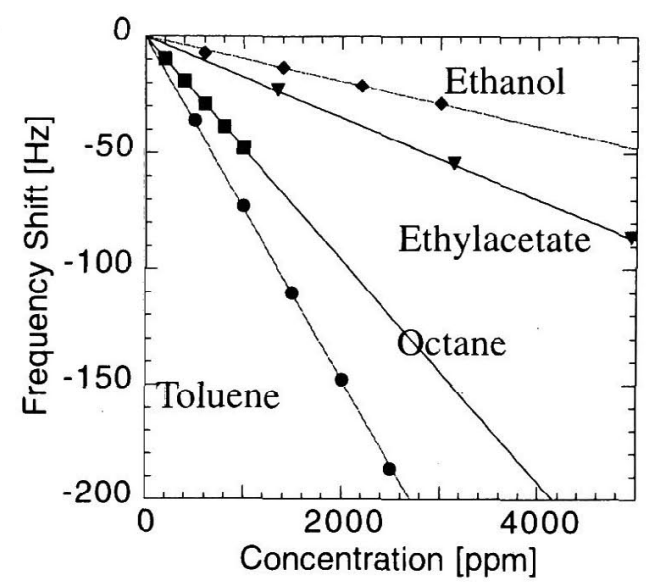

Figure 6. Frequency shift of a resonant beam gas sensor as a function of different concentrations of various analytes. The gas sensor was covered with 2.4 um of PEUT [12].

well membrane with 300 thermocouples covered by the chemically sensitive polymer [13]. Enthalpy changes during absorption and desorption of gaseous analytes into the polymer cause (transient) temperature variations of the thermally insulated micromachined membrane. Polysilicon/aluminum thermopiles are used to measure these temperature variations. A polymer coated sensing membrane and an uncoated reference membrane are connected in parallel to the input stage of a low-noise amplifier to measure the temperature differences between the two membranes.

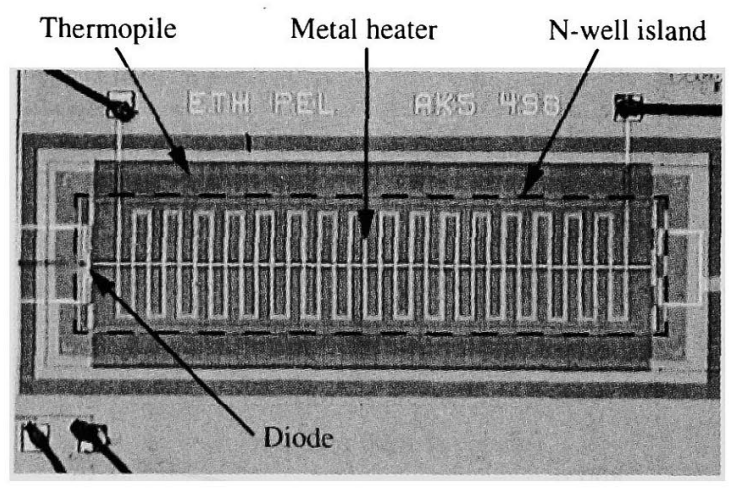

Figure 7. Micrograph of the calorimetric chemical sensor with a rectangular $n$-well island structure, thermopile, metal heater, and on-membrane diode [13].

Fig. 8 shows the block diagram of the low-noise chopper-stabilized instrumentation amplifier that has been designed to measure the weak sensor signals. In order to avoid the large $1 / \mathrm{f}$-noise in CMOS input stages, the input signals are first mixed to higher frequencies by the input chopper. Then, the signal is amplified by a three stage amplifier and finally demodulated by the output chopper. The bandpass filter after the first amplification stage avoids saturation of the system by the DC-offset of the first amplifier and reduces the residual offset caused by demodulated charge injection from the input chopper [14]. The amplifier features a gain of $75 \mathrm{~dB}$, a bandwidth of $500 \mathrm{~Hz}$, and an equivalent input noise of $12 \mathrm{nV} / \sqrt{\mathrm{Hz}}$. The equivalent residual input offset was measured to be smaller than $1 \mu \mathrm{V}$. 


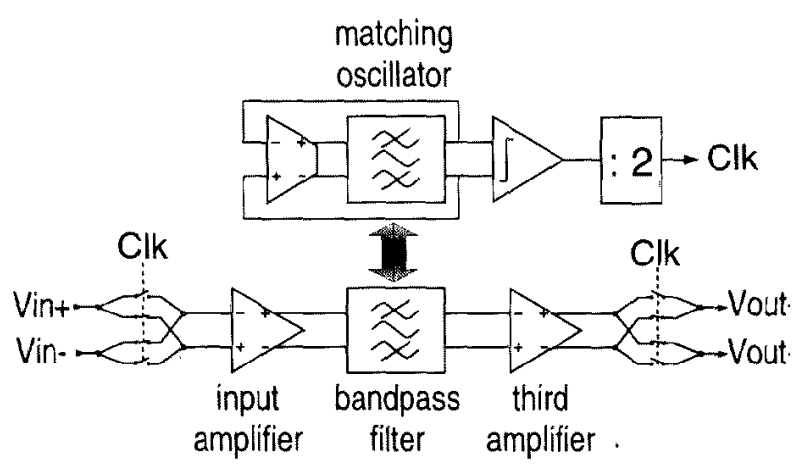

Figure 8. Block diagram of low-noise chopper amplifier [14]

In contrast to the capacitive and gravimetric chemical sensors, the calorimetric sensor detects transient (non-equilibrium) signals only, i.e., changes in the analyte concentration. Figs. 9 (a) and (b) show the output voltage of the microsystem while switching $1500 \mathrm{ppm}$ of toluene in air on and off, respectively, at $33 \mathrm{sccm}$ flow rate and a temperature of $28^{\circ} \mathrm{C}$ [13]. The sensing membrane was coated with a $10 \mu \mathrm{m}$ thick poly(etherurethane) layer. The enthalpy changes are calculated by integrating the sensor output signal and calibration using a known heating power dissipated in a metal heating resistor located on the membranes. The resulting enthalpy changes of about $30 \mu \mathrm{J}$ are identical for switching on and off the analyte.

(a)

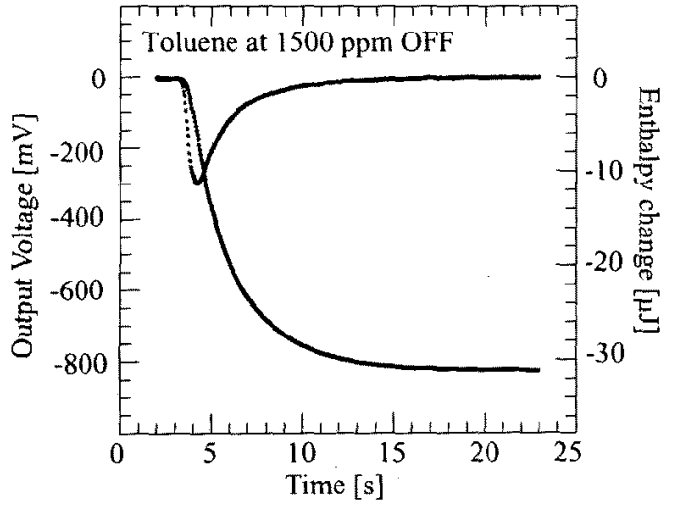

(b)

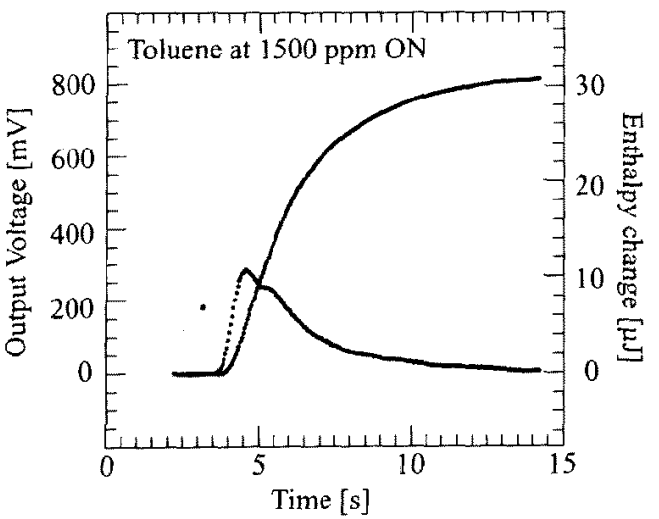

Figure 9. Output voltage of the calorimetric chemical sensor system while switching $(a)$ off and (b) on 1500 ppm of toluene in air at $28.0^{\circ} \mathrm{C}$. The resulting enthalpy change was calculated by integrating the sensor output signal and calibration using the integrated heating resistor [13].
The authors are greatly indebted to current and former staff of the Physical Electronics Laboratory at ETH Zurich, notably A. Hierlemann, Prof. S. Kawahito (Shizuoka University, Japan), N. Kerness, A. Koll, D Lange, and T. Müller. The excellent services of the prototype manufacturer Austria Mikro Systeme International are gratefully acknowledged.

This work is supported by the Körber Foundation, Germany, and the Swiss Priority Program MINAST.

(Manuscript received Feb. 21, 2000)

\section{REFERENCES}

[1] Information on the MOSES II system can be found on the following WWW-pages; http://www.lennartz-electronic.de and http://www.motech.de.

[2] K. D. Wise (ed.), "Special Issue: Integrated Sensors, Microactuators \& Microsystems (MEMS)," Proc. IEEE, vol. 86, no. $8,1998$.

[3] H. Baltes, O. Paul, O. Brand, "Micromachined thermallybased CMOS microtransducers," Proc. IEEE, vol. 86, pp. 1660-1678, 1998.

[4] A. Schaufelbühl, N. Schneeberger, U. Münch, O. Paul, H. Baltes, C. Menolfi, Q. Huang, "Uncooled low-cost thermal imager using micromachined CMOS integrated sensor array," Transducers 99 Digest of Technical Papers, 1999, pp. 606-609.

[5] C. Hierold, "Intelligent CMOS sensors," Proc. IEEE Int Conf. on Micro Electro Mechanical Systems, 2000, pp. 1-6.

[6] A. Hierlemann, A. Koll, D. Lange, C. Hagleitner, N. Kerness, O. Brand, H. Baltes, "CMOS-based chemical microsensors: components of a micronose system," Proc. SPIE, vol. 3857, pp. 158-169, 1999

[7] T. Müller, T. Feichtinger, G. Breitenbach, M. Brandl, O. Brand, H. Baltes, "Industrial fabrication method for arbitrarily shaped silicon n-well micromechanical structures," Proc. IEEE Workshop on Micro Electro Mechanical Systems MEMS 98, 1998, pp. 240-245.

[8] A. Koll, S. Kawahito, F. Mayer, C. Hagleitner, D. Scheiwiller, O. Brand, H. Baltes, "A flip-chip packaged CMOS chemical microsystem for detection of volatile organic compounds," Proc. SPIE, vol. 3328, pp. 223-232, 1998.

[9] A. Koll, A. Schaufelbühl, N. Schneeberger, U. Münch, O. Brand, H. Baltes, C. Menolfi, Q. Huang, "Micromachined CMOS calorimetric chemical sensor with on-chip low noise amplifier," Proc. IEEE Workshop on Micro Electro Mechanical Systems MEMS 99, 1999, pp. 547-551.

[10] C. Hagleitner, A. Koll, R. Vogt, O. Brand, H. Baltes, "CMOS capacitive chemical microsystem with active temperature control for discrimination of organic vapors," Transducers 99 Digest of Technical Papers, 1999, pp. 1012-1015.

[11] S. Kawahito, A. Koll, C. Hagleitner, H. Baltes, Y. Tadokoro, "Delta-sigma modulation sensor interface circuits with improved conversion gain for capacitive readout chemical sensors", Trans. IEE Japan, vol 119-E, pp. 138-142, 1999.

[12] D. Lange, C. Hagleitner, O. Btand, H. Baltes, "CMOS resonant beam gas sensor with integrated preamplifier," Transducers 99 Digest of Technical Papers, 1999, pp. 1020-1023.

[13] N. Kerness, A. Koll, A. Schaufelbühl, C. Hagleitner, A. Hierlemann, O. Brand, H. Baltes, "N-well based CMOS calorimetric chemical sensor," Proc. IEEE Int. Conf. on Micro Electro Mechanical Systems, 2000, pp. 96-101.

[14] C. Menolfi, Q. Huang, "A low noise CMOS instrumentation amplifier for thermoelectric infrared detectors," IEEE $J$. Solid State Circ, vol. 32, pp. 1-9, 1997. 
Henry Baltes received the D Sc degree from ETH Zurich in 1971.

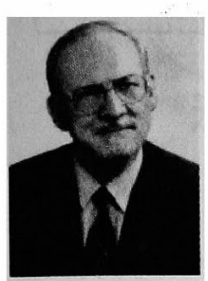

In the following years he was on the faculty of the Freie Universität Berlin and the University of Düsseldorf, Germany, the University of Waterloo, Ontario, Canada, and EPF Lausanne, Switzerland. From 1974 to 1982 he worked for Landis \& Gyr Zug, Switzerland, where he directed the solid-state device laboratory. From 1975 to 1982 , he taught at EPF Lausanne.

From 1983 to 1988, he held the Henry Marshall Tory Chair at the University of Alberta, Edmonton, Canada, where he directed a research program in microsensors. As acting President of the Alberta Microelectronic Centre he was in charge of establishing a CMOS metallization fab. From 1986 to 1988, he was a Director of LSI Logic Corporation of Canada.

Since 1988 he is Professor of Physical Electronics at ETH Zurich and Director of the Physical Electronics Laboratory active in silicon integrated microsystems. From 1992 to 1996 he was the Director of the Swiss national priority research program LESIT. In 1996, he was Visiting Professor at Stanford University and the University of Waterloo. In the same year he became Member of the Swiss Academy of Technical Sciences. In 1998 he received the Koerber-Award and became a co-founder of the spin-off company Sensirion (formerly Alpha Sensors).

Professor Baltes is Editor of Sensors Update (Germany), Member of the Institute of Electrical and Electronic Engineers, Optical Society of America, Electrochemical Society, Institute of Physics (London), Swiss Academy of Technical Sciences, Swiss Management Association, Swiss Society for Sensor Technology, Swiss Information Technology Society, Swiss Electrotechnical Association, and Swiss Physical Society. He authored $400+$ technical publications, 15 patents, and 5 books.

Oliver Brand received the diploma degree in physics from Tech-

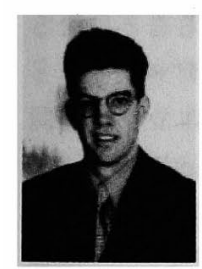
nical University Karlsruhe, Germany, and the D. Sc. degree from ETH Zurich (Swiss Federal Institute of Technology), Switzerland, in 1990 and 1994, respectively.

From 1995 until 1997, he was a Postdoctoral Fellow with the Georgia Institute of Technology, Atlanta, USA. In 1997, he joined the Physical Electronics Laboratory of ETH Zurich as Project Leader, Group Leader and Lecturer. His research interest focuses on the development of micro- and nanosensors using industrial IC processes in combination with post-processing micromachining steps and dedicated packaging technologies.

Christoph Hagleitner received his diploma in electrical engineering in 1997 from ETH Zurich., Switzerland

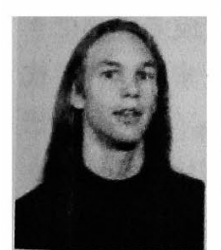
where he is currently working towards his Ph.D. degree. His research interests are interface circuitry for smart sensor systems in CMOS technology and parallel scanning atomic force microscopy. 